Network Working Group

Request for Comments: 2128

Category: Standards Track
G. Roeck, Editor

cisco Systems

March 1997

\title{
Dial Control Management Information Base using SMIv2
}

Status of this Memo

This document specifies an Internet standards track protocol for the Internet community, and requests discussion and suggestions for improvements. Please refer to the current edition of the "Internet Official Protocol Standards" (STD 1) for the standardization state and status of this protocol. Distribution of this memo is unlimited.

\section{Abstract}

This memo defines a portion of the Management Information Base (MIB) for use with network management protocols in the Internet community. In particular, it describes managed objects used for managing demand access circuits, including ISDN.

This document specifies a MIB module in a manner that is compliant to the SNMPV2 SMI. The set of objects is consistent with the SNMP framework and existing SNMP standards.

This document is a product of the ISDN MIB working group within the Internet Engineering Task Force. Comments are solicited and should be addressed to the working group's mailing list at isdnmibecisco.com and/or the author.

Table of Contents

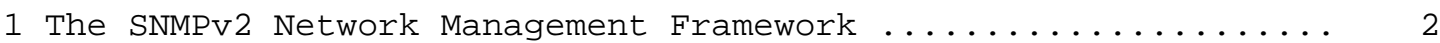

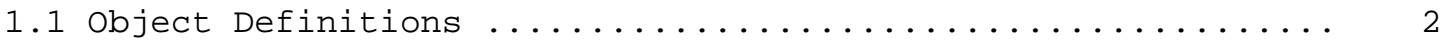

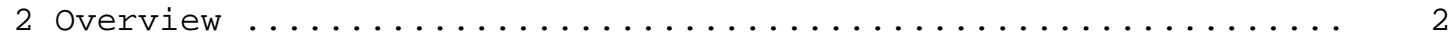

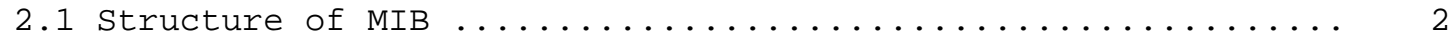

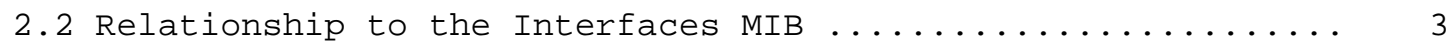

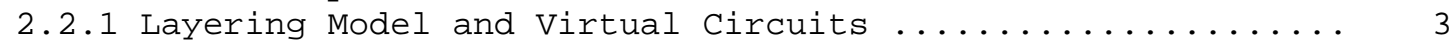

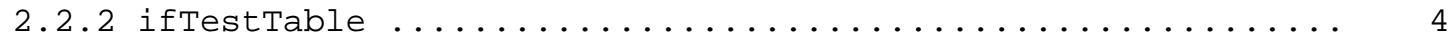

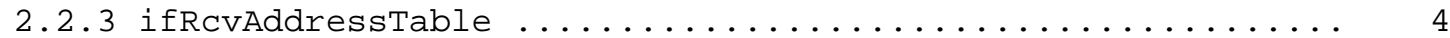

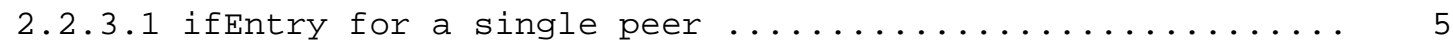

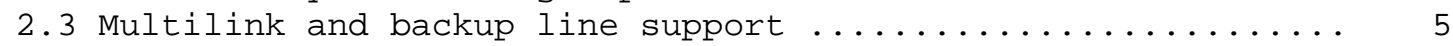

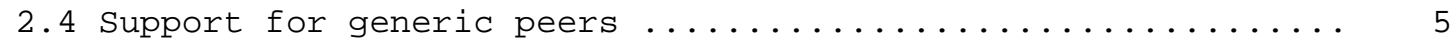

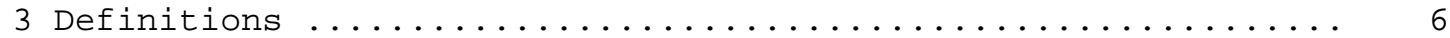

3.1 Dial Control MIB .......................... 6

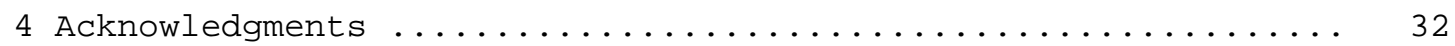

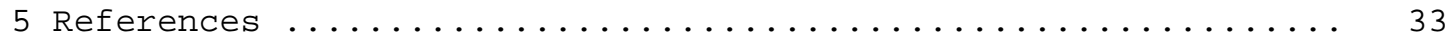




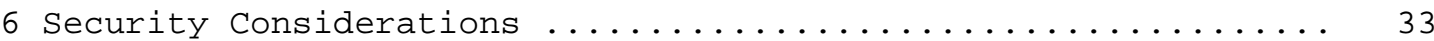

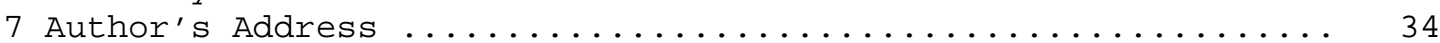

1. The SNMPv2 Network Management Framework

The SNMPv2 Network Management Framework presently consists of three major components. They are:

- the SMI, described in RFC 1902 [1] - the mechanisms used for describing and naming objects for the purpose of management.

o the MIB-II, STD 17, RFC 1213 [2] - the core set of managed objects for the Internet suite of protocols.

o the protocol, STD 15, RFC 1157 [3] and/or RFC 1905 [4], the protocol for accessing managed objects.

The Framework permits new objects to be defined for the purpose of experimentation and evaluation.

\subsection{Object Definitions}

Managed objects are accessed via a virtual information store, termed the Management Information Base or MIB. Objects in the MIB are defined using the subset of Abstract Syntax Notation One (ASN.1) defined in the SMI. In particular, each object type is named by an OBJECT IDENTIFIER, an administratively assigned name. The object type together with an object instance serves to uniquely identify a specific instantiation of the object. For human convenience, we often use a textual string, termed the descriptor, to refer to the object type.

\section{Overview}

\subsection{Structure of MIB}

Managing demand access circuits requires the following groups of information:

- General configuration information.

- Information to describe peer configuration and peer statistics. In this respect, peer configuration means information on how to connect to peers on outgoing calls, how to identify peers on incoming calls, and other call related configuration information.

- Information to store active call information. 
○

Information to retain call history.

The MIB, therefore, is structured into four groups.

- The dialCtlConfiguration group is used to specify general configuration information.

- The dialctlpeer group is used to describe peer configuration and peer statistics.

- The callActive group is used to store active call information.

- The callHistory group is used to store call history information. These calls could be circuit switched or they could be virtual circuits. History of each and every call is stored, of successful calls as well as unsuccessful and rejected calls. An entry will be created when a call is cleared.

2.2. Relationship to the Interfaces MIB

This section clarifies the relationship of this MIB to the Interfaces MIB [8]. Several areas of correlation are addressed in the following subsections. The implementor is referred to the Interfaces MIB document in order to understand the general intent of these areas.

\subsubsection{Layering Model and Virtual Circuits}

On an occasional access channel, there are a number of peer systems that are permitted to call or be called, all of which need to be treated as active from a routing viewpoint, but most of which have no call in progress at any given time.

On dialup interfaces, this is further complicated by the fact that calls to a given peer float from channel to channel. One cannot definitively say "I call this peer on that interface." It is necessary, therefore, to provide a mapping algorithm between the low-level interfaces, and the various logical interfaces supporting the peers. This is solved by creating a logical interface (ifEntry) for each peer and a logical interface (ifEntry) for each low-level interface. These are then correlated using the ifstackTable.

The low-level interfaces are either physical interfaces, e.g. modem interfaces, or logical interfaces, e.g. ISDN B channels, which then in turn are layered on top of physical ISDN interfaces. 
The model, therefore, looks something like this, taking ISDN as an example:

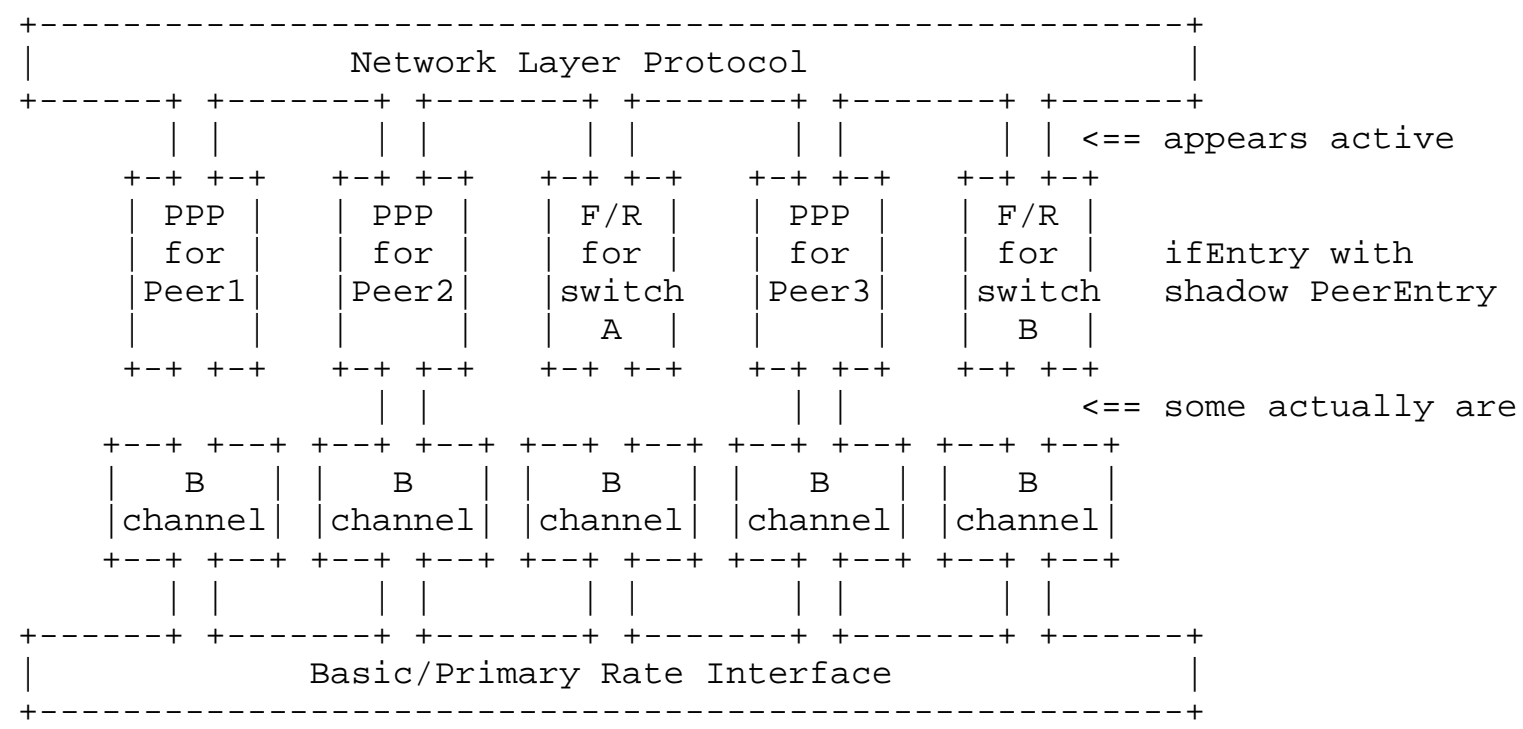

Mapping of IP interfaces to Called Peers to B Channels

IfEntries are maintained for each peer.

In this model, each peer is required to have an associated encapsulation layer interface. This interface can be of any kind, e.g. PPP or LAPB.

In order to specify the network address for a given peer, one would then usually add a routing/forwarding table entry, pointing to the encapsulation layer interface through which this peer can be reached.

\subsection{2. ifTestTable}

The ifTestTable usage is defined in the MIBs defining the encapsulation below the network layer. For example, if PPP encapsulation is being used, the ifTestTable is defined by PPP.

\subsection{3. ifRcvAddressTable}

The ifRcvAddressTable usage is defined in the MIBs defining the encapsulation below the network layer. For example, if PPP encapsulation is being used, the ifRcVAddressTable is defined by PPP. 


\subsubsection{1. ifEntry for a single peer}

IfEntries are defined in the MIBs defining the encapsulation below the network layer. For example, if PPP encapsulation is being used, the ifEntry is defined by PPP.

ifEntries will never be created by the Dial Control MIB. The Dial Control MIB always depends on some other ifIndex of some set of iftypes. That is, to create an entry in the Dial Control MIB, the base ifEntry must already have been created through some other mechanism.

The Dial Control entry does have its own RowStatus, permitting the Dial Control supplementary information to come and go, but not otherwise disturbing the ifIndex to which it is attached. If in a given implementation the two are tightly bound, deleting the ifEntry may have the side effect of deleting the Dial Control entry.

2.3. Multilink and backup line support

In order to support multilink and backup procedures, there may be several entries for a single peer in the dialctlPeercfgTable.

A single peer is identified using the dialctlpeercfgld object of the dialctlpeerCfgTable. There may be several entries in dialctlpeerCfgTable with the same value of dialctlpeerCfgId, but different ifIndex values. Each of those entries will then describe a possible connection to the same peer. Such entries can then be used to handle multilink as well as backup procedures, e.g. by bundling the attached ifEntries using PPP multilink.

\subsection{Support for generic peers}

Generic peers can for example be supported by permitting wild-card characters (e.g.,' '?' or '*') in dialctlPeerCfgAnswerAddress. A number to be accepted could then be defined as partly (e.g.,' '*1234') or entirely generic (e.g.,,$\star \prime \prime)$.

A detailed specification of such a functionality is outside the scope of this document.

However, the implementor should be aware that supporting generic peers may cause a security hole. The user would not know where a call is from, which could potentially allow unauthorized access. 
3. Definitions

3.1. Dial Control MIB

DIAL-CONTROL-MIB DEFINITIONS : := BEGIN

IMPORTS

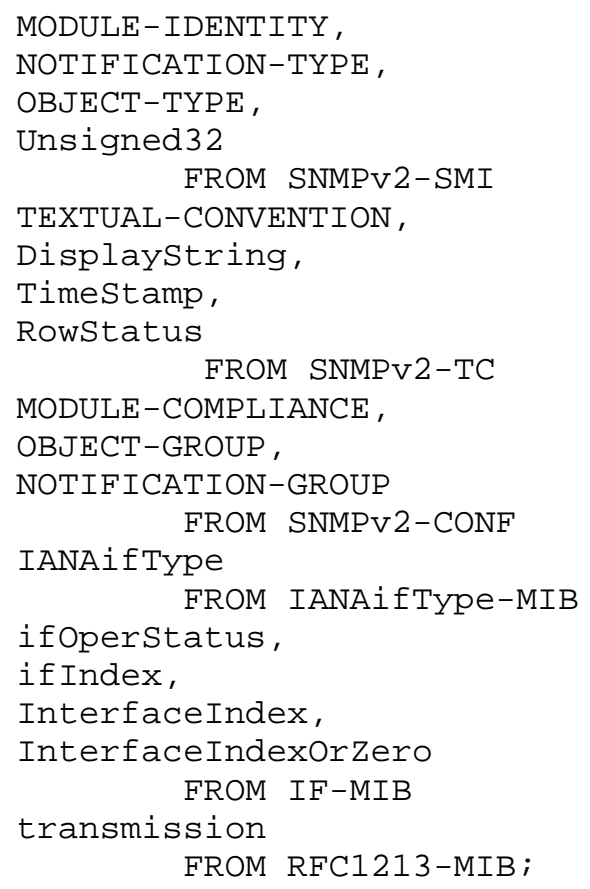




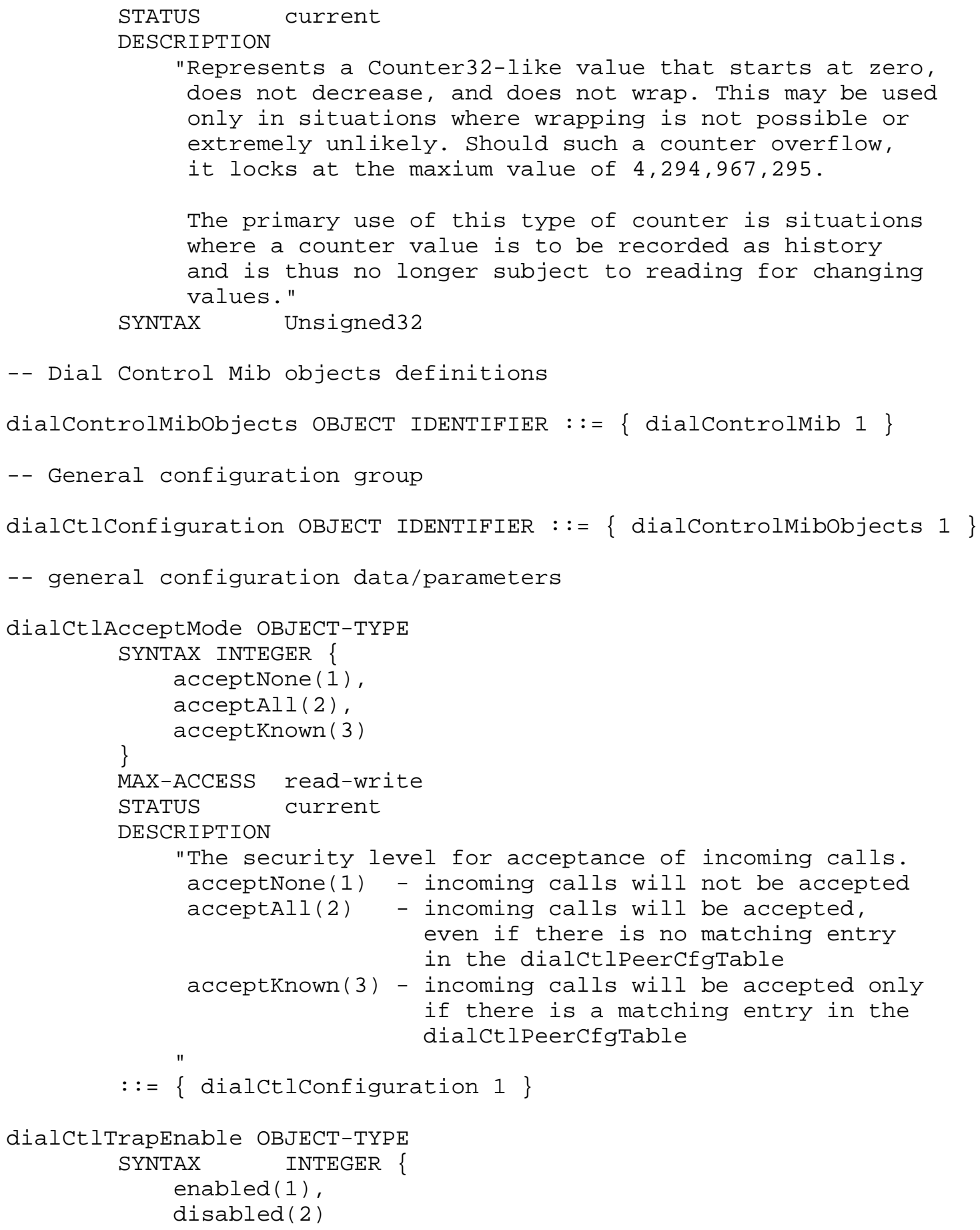

Roeck 
$::=\{$ dialCtipeerCfgTable 1$\}$

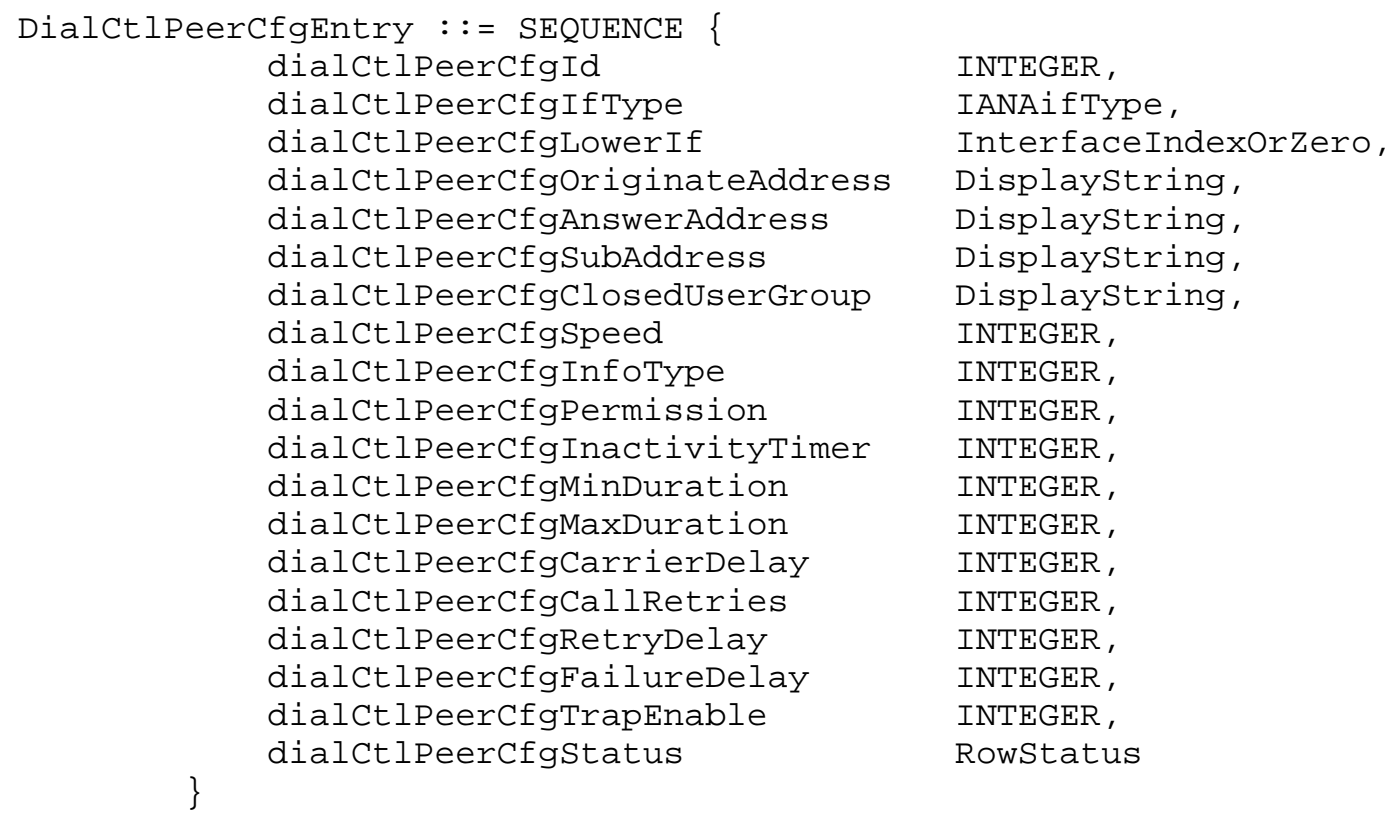

dialctlPeerCfgId OBJECT-TYPE

SYNTAX INTEGER (1..2147483647)

MAX-ACCESS not-accessible

STATUS current

DESCRIPTION

"This object identifies a single peer. There may

be several entries in this table for one peer,

defining different ways of reaching this peer.

Thus, there may be several entries in this table

with the same value of dialctlpeercfgid.

Multiple entries for one peer may be used to support multilink as well as backup lines.

A single peer will be identified by a unique value of this object. Several entries for one peer MUST

have the same value of dialctlPeerCfgId, but different ifEntries and thus different values of ifIndex."

$::=\{$ dialctlpeerCfgEntry 1$\}$

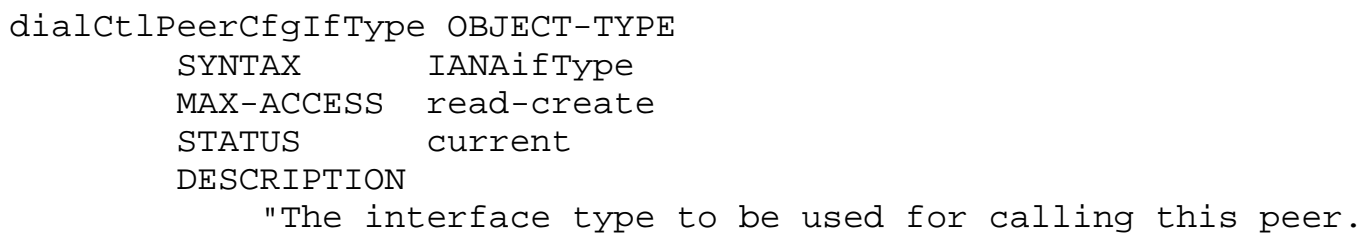




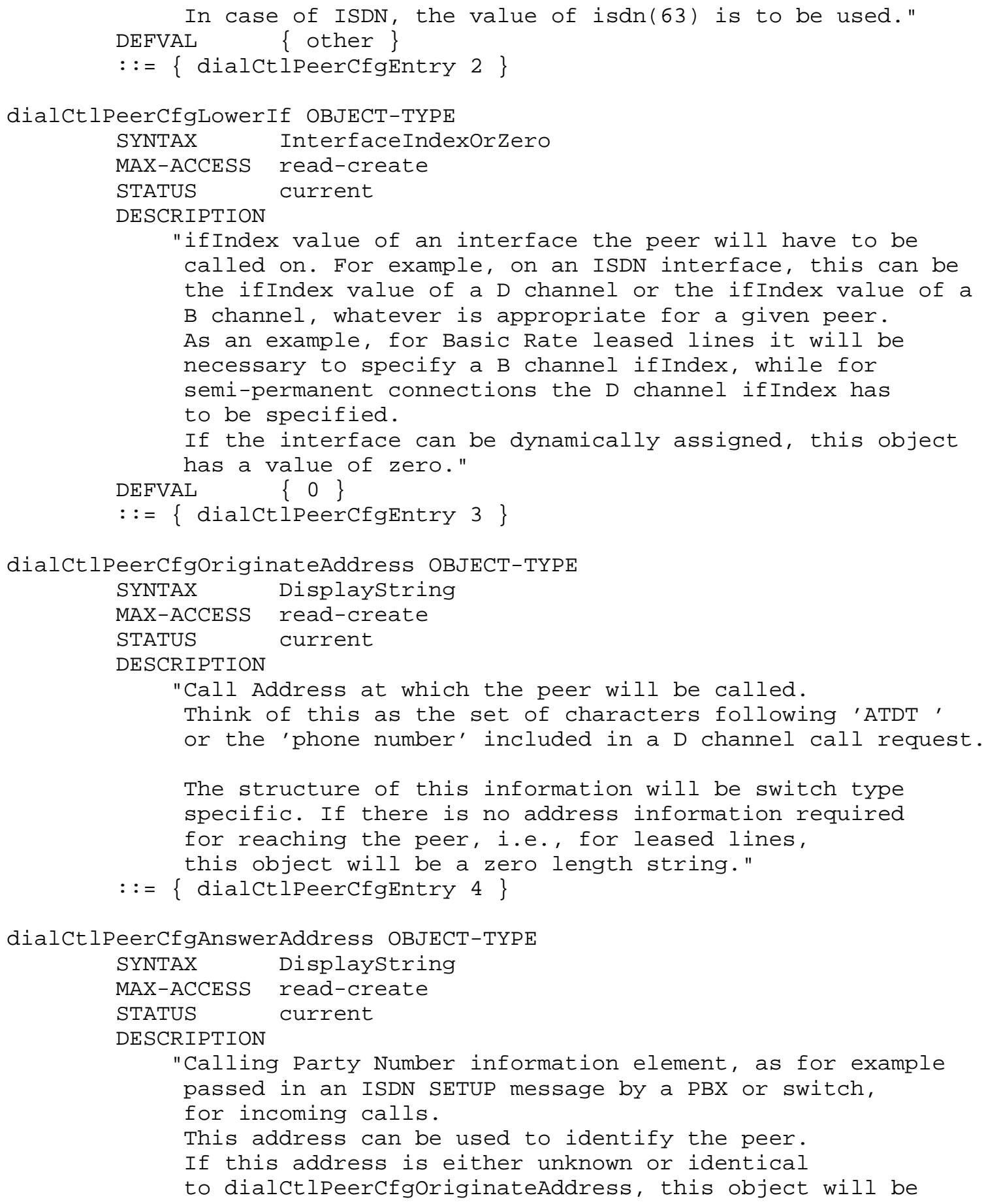

Roeck 


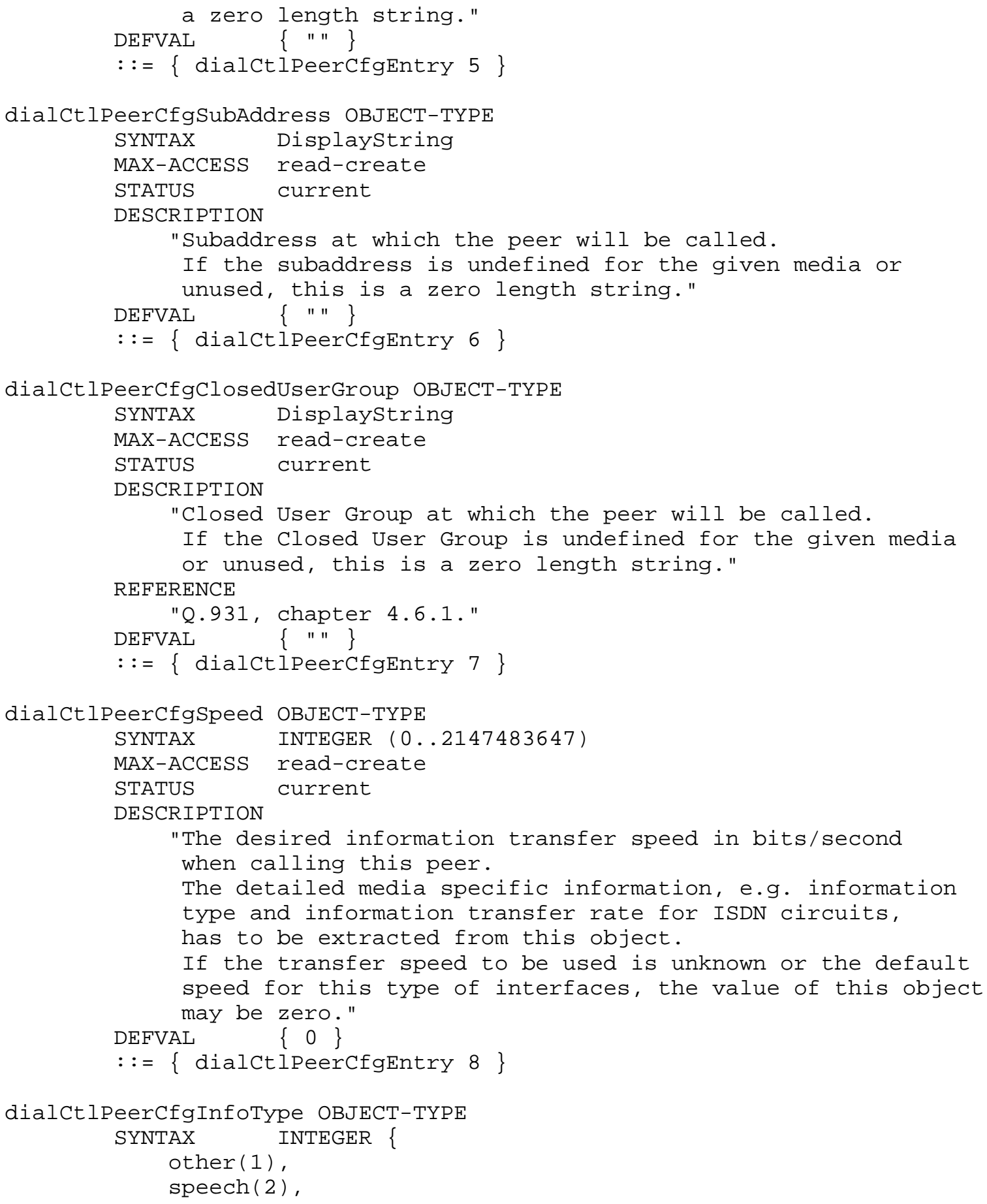




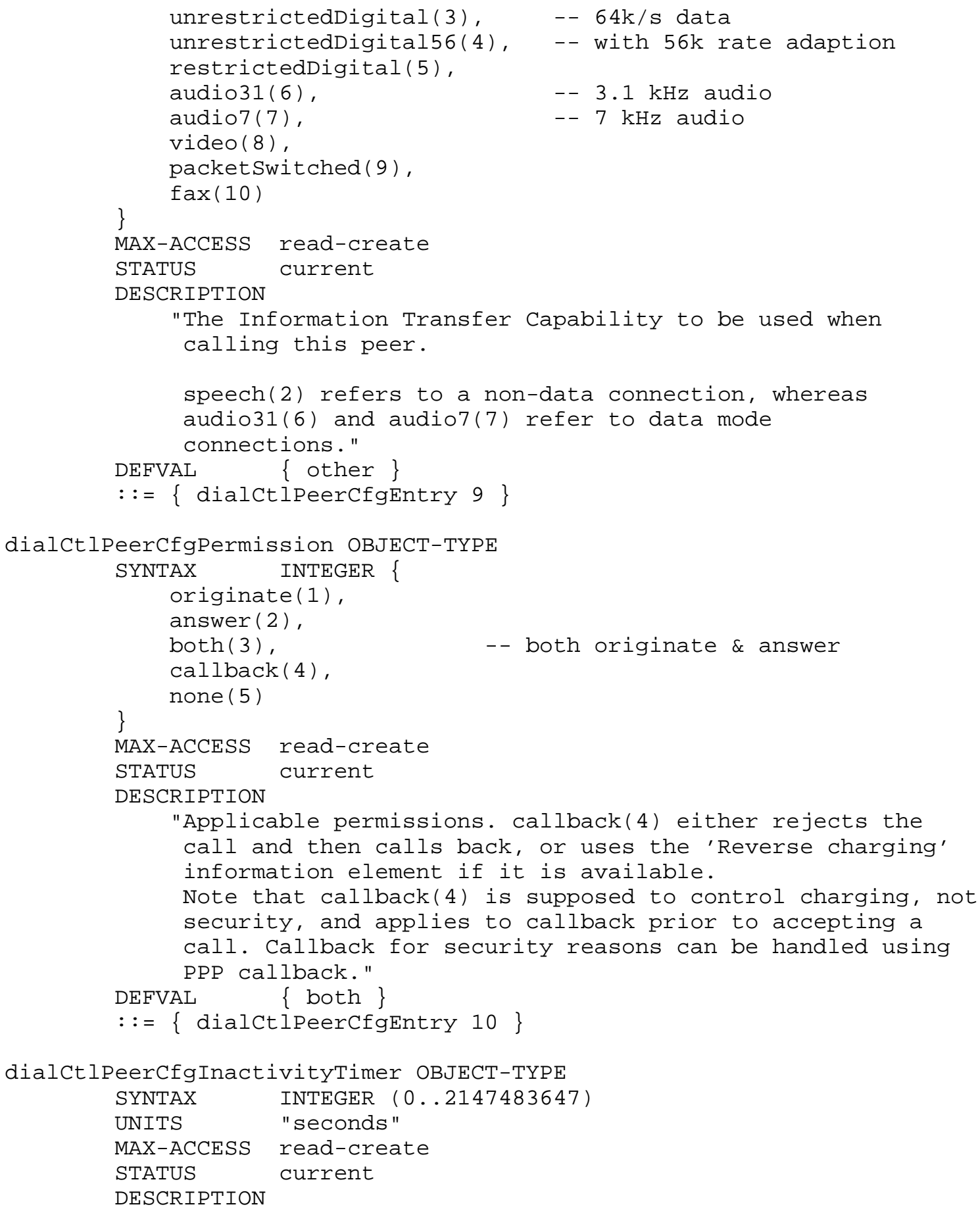




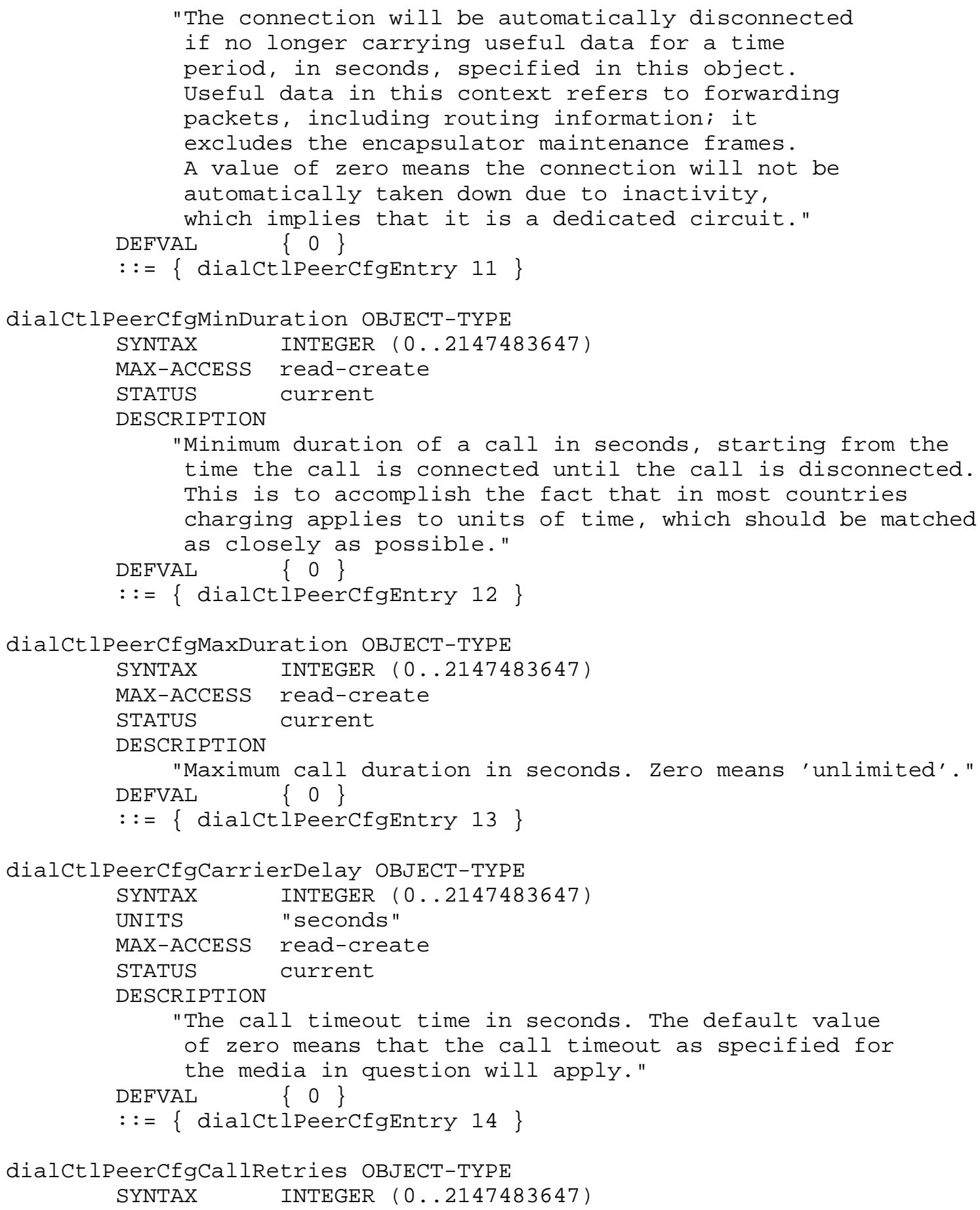

Roeck 


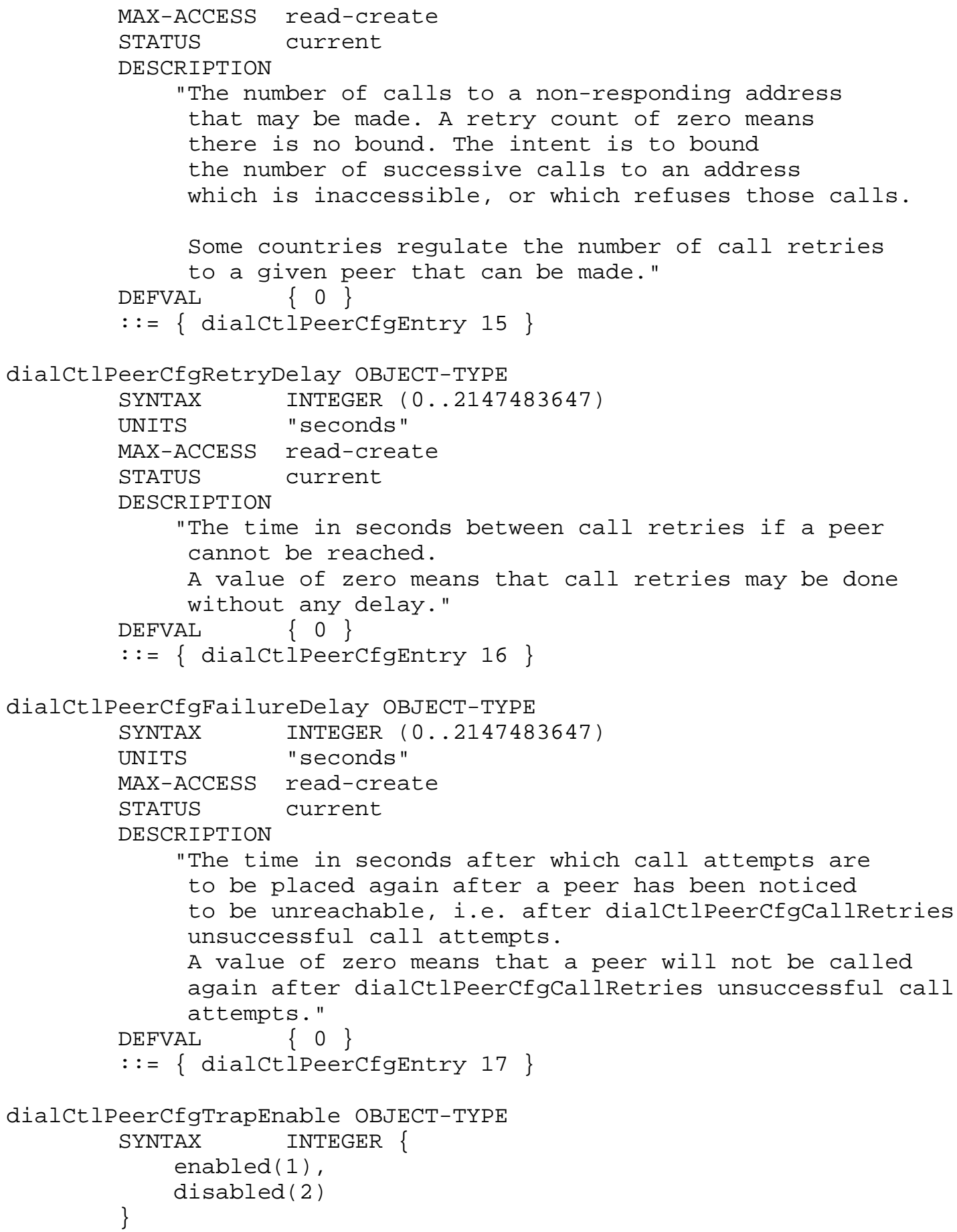

Roeck 


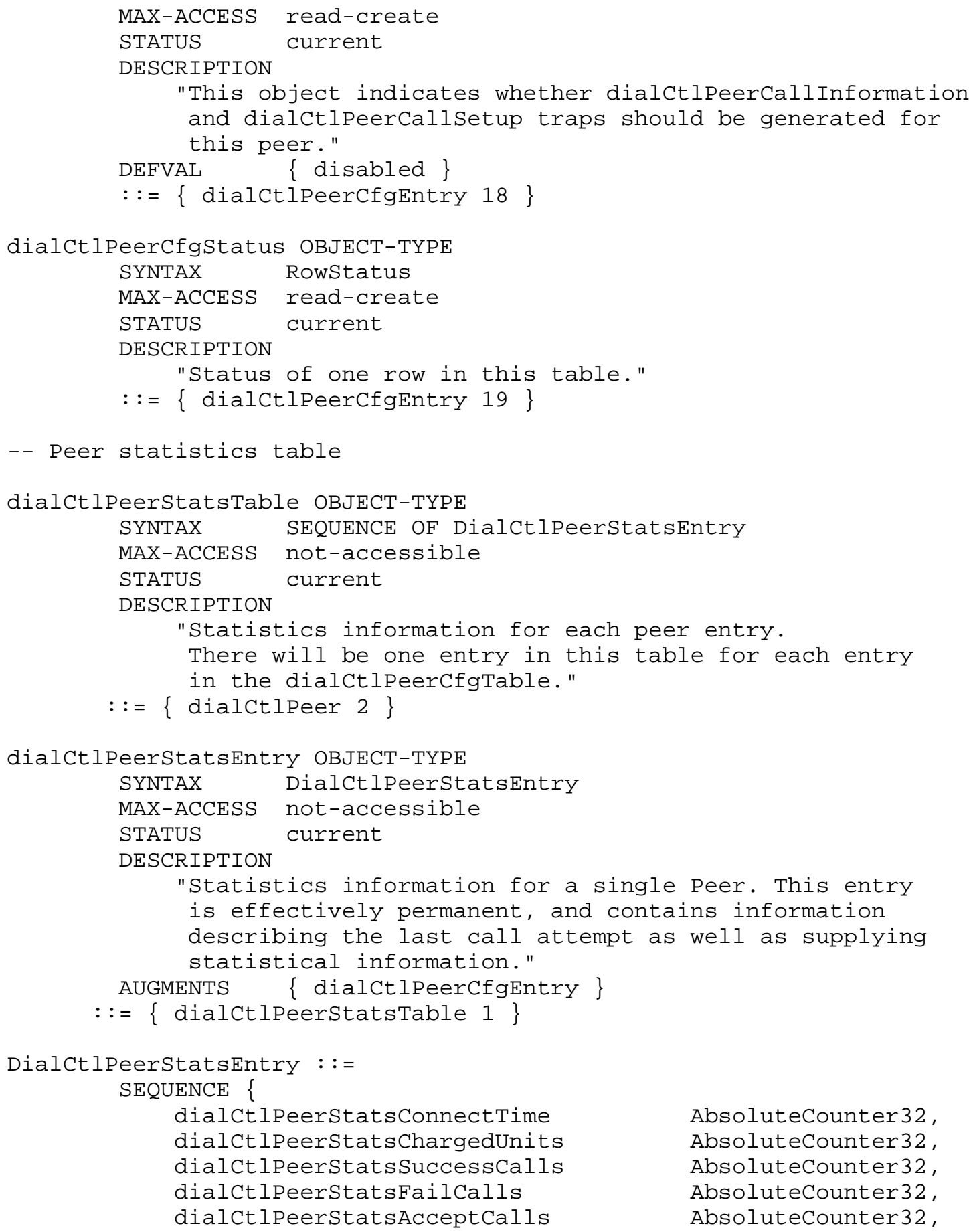

Absolutecounter32, Absolutecounter32, Absolutecounter32, Absolutecounter32, AbsoluteCounter32,

Roeck 


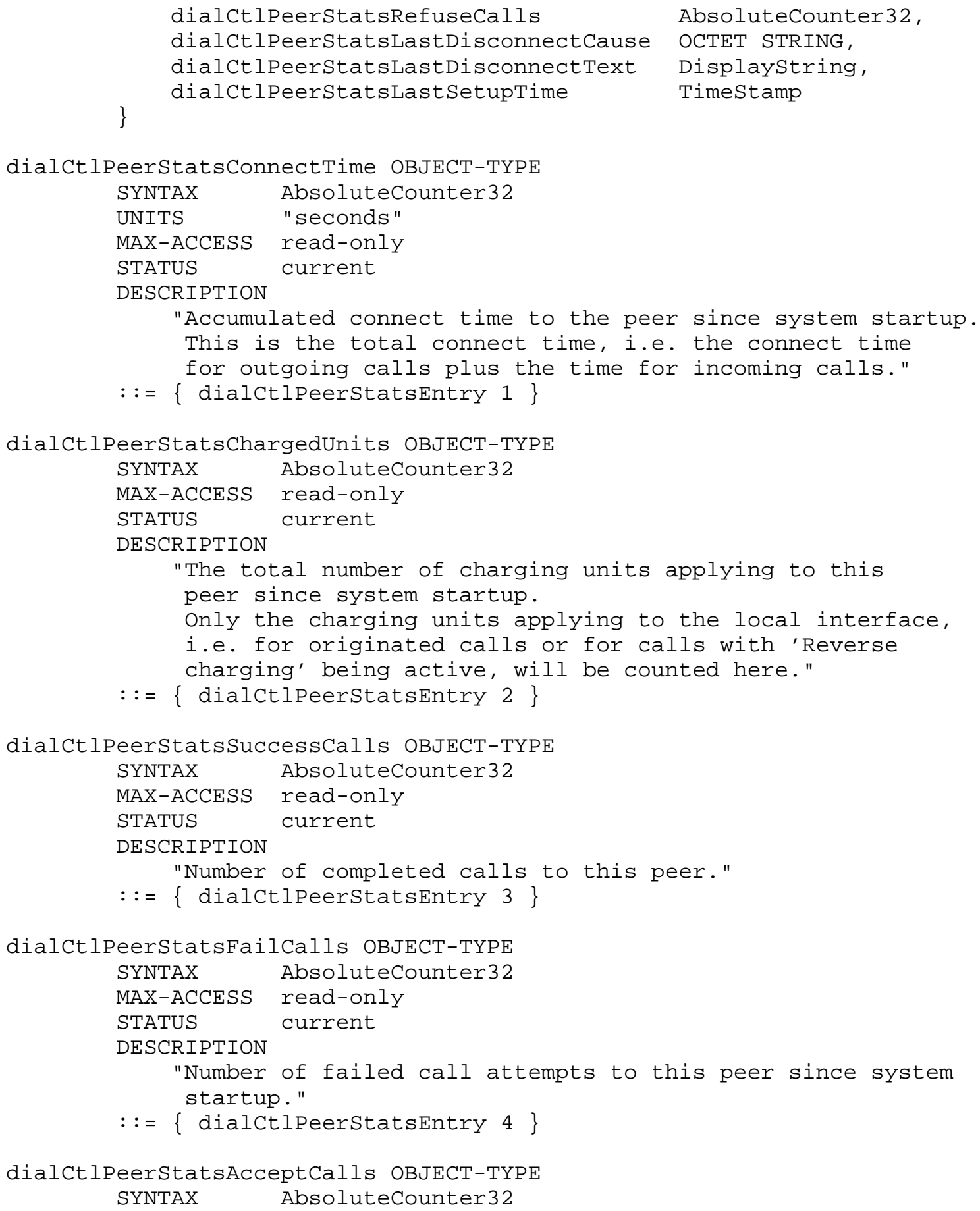

Roeck 


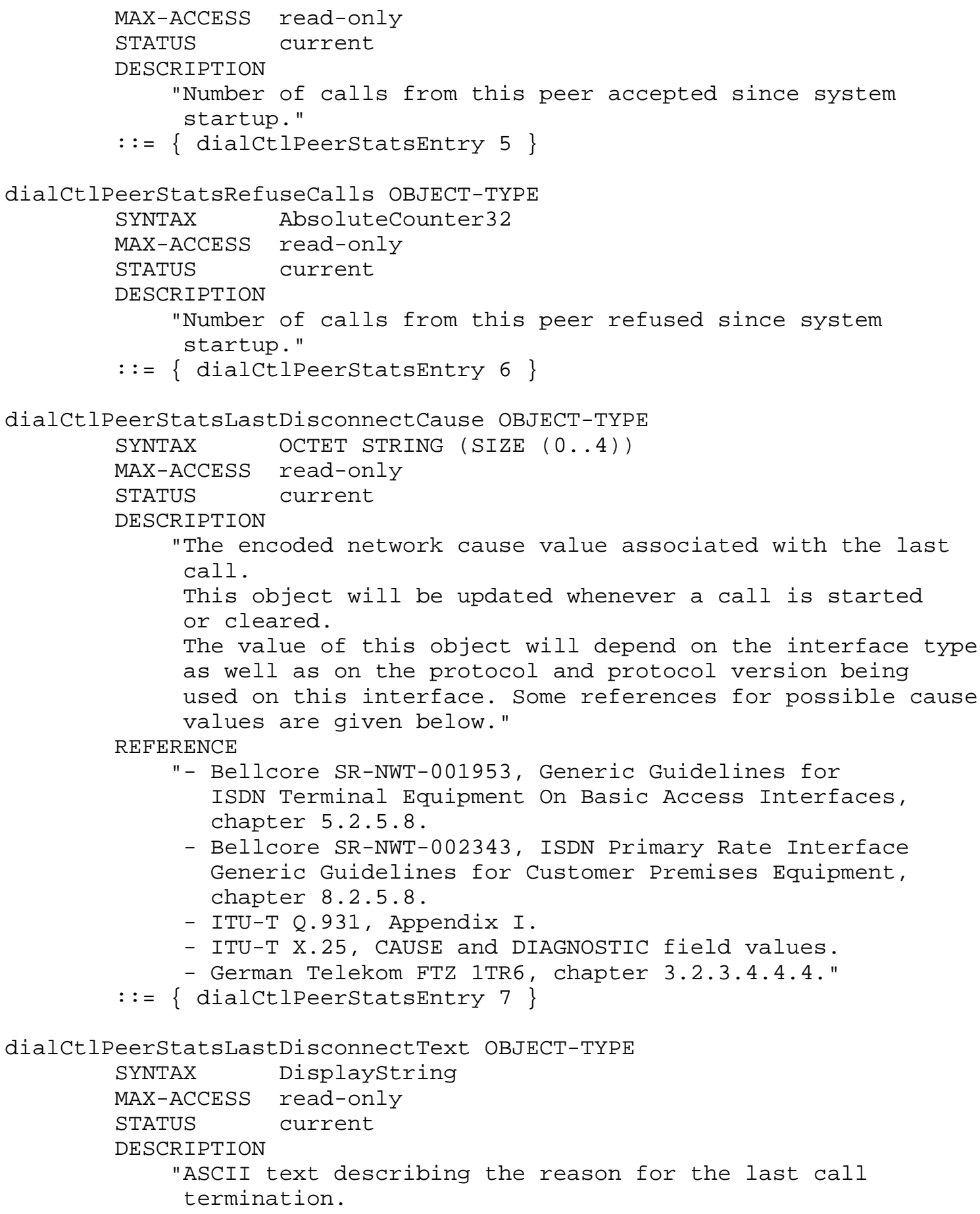

Roeck 


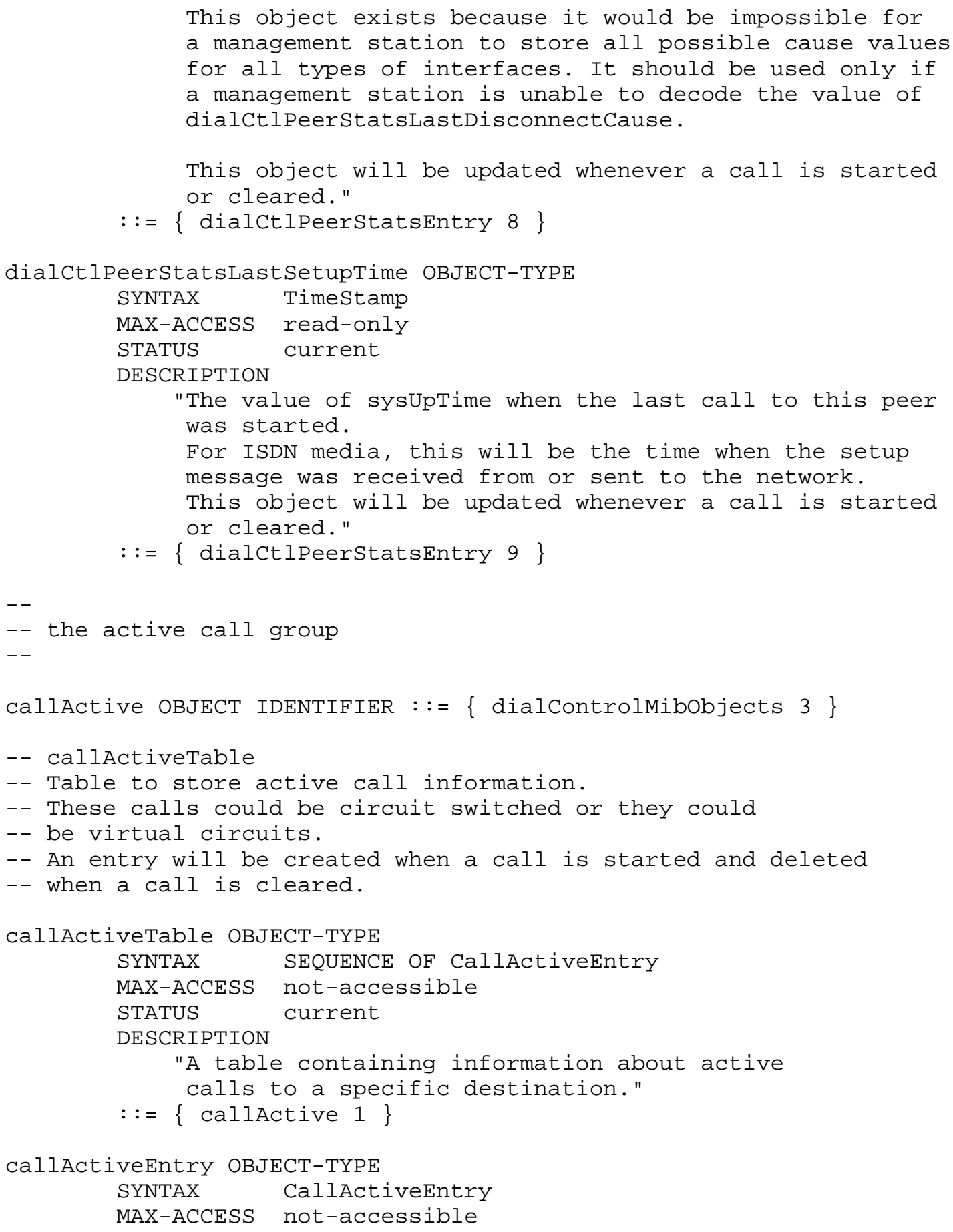




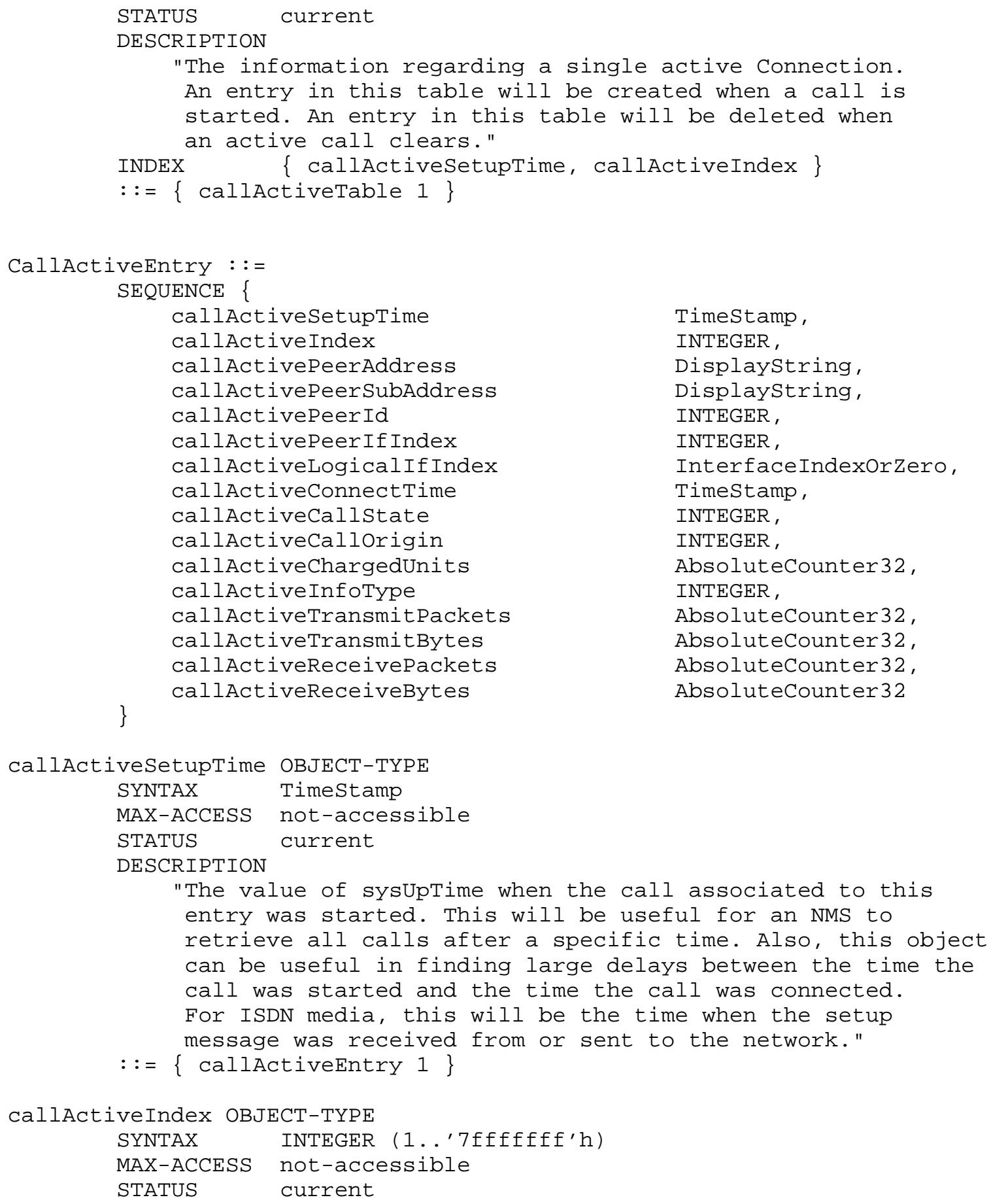




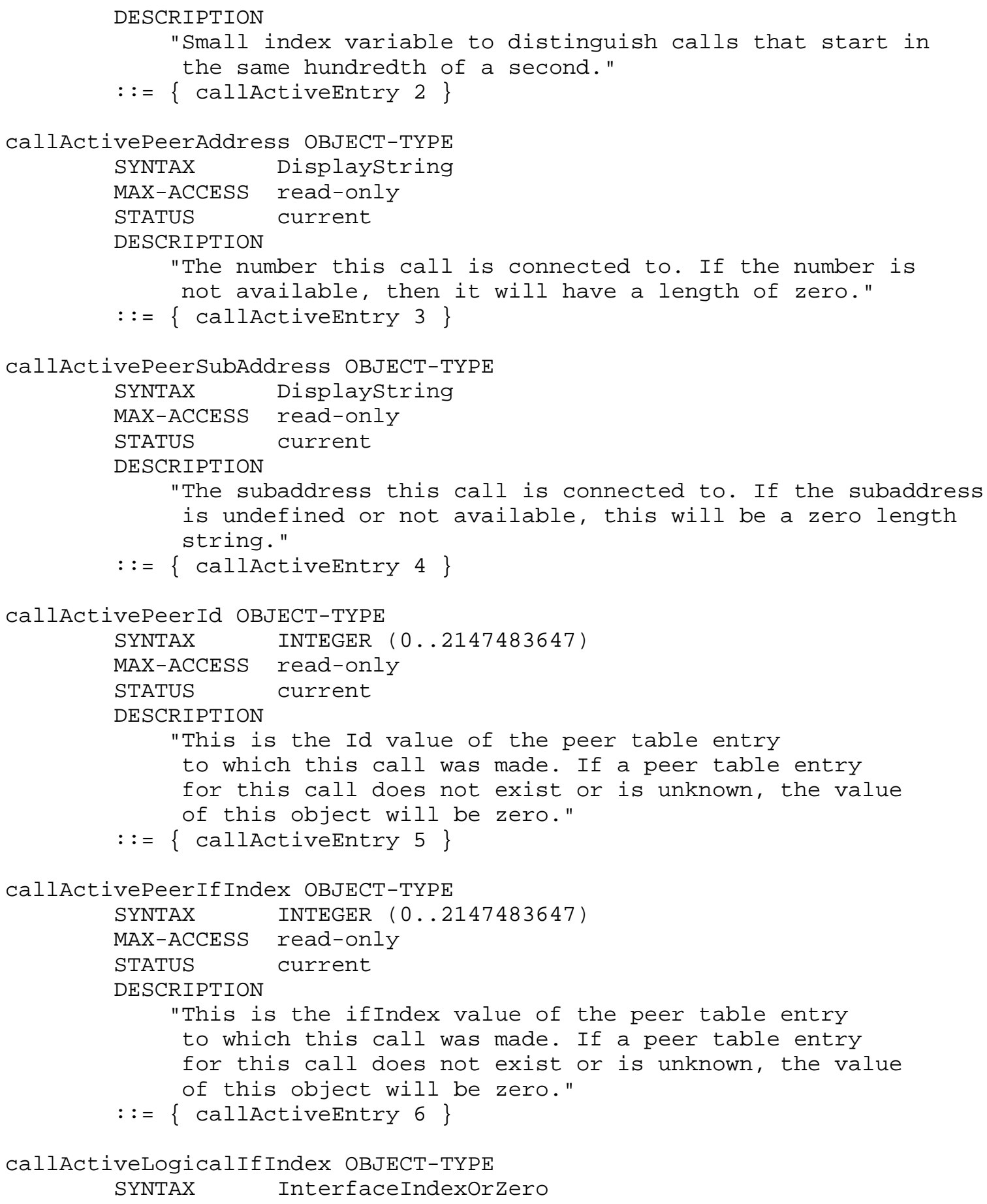

Roeck 


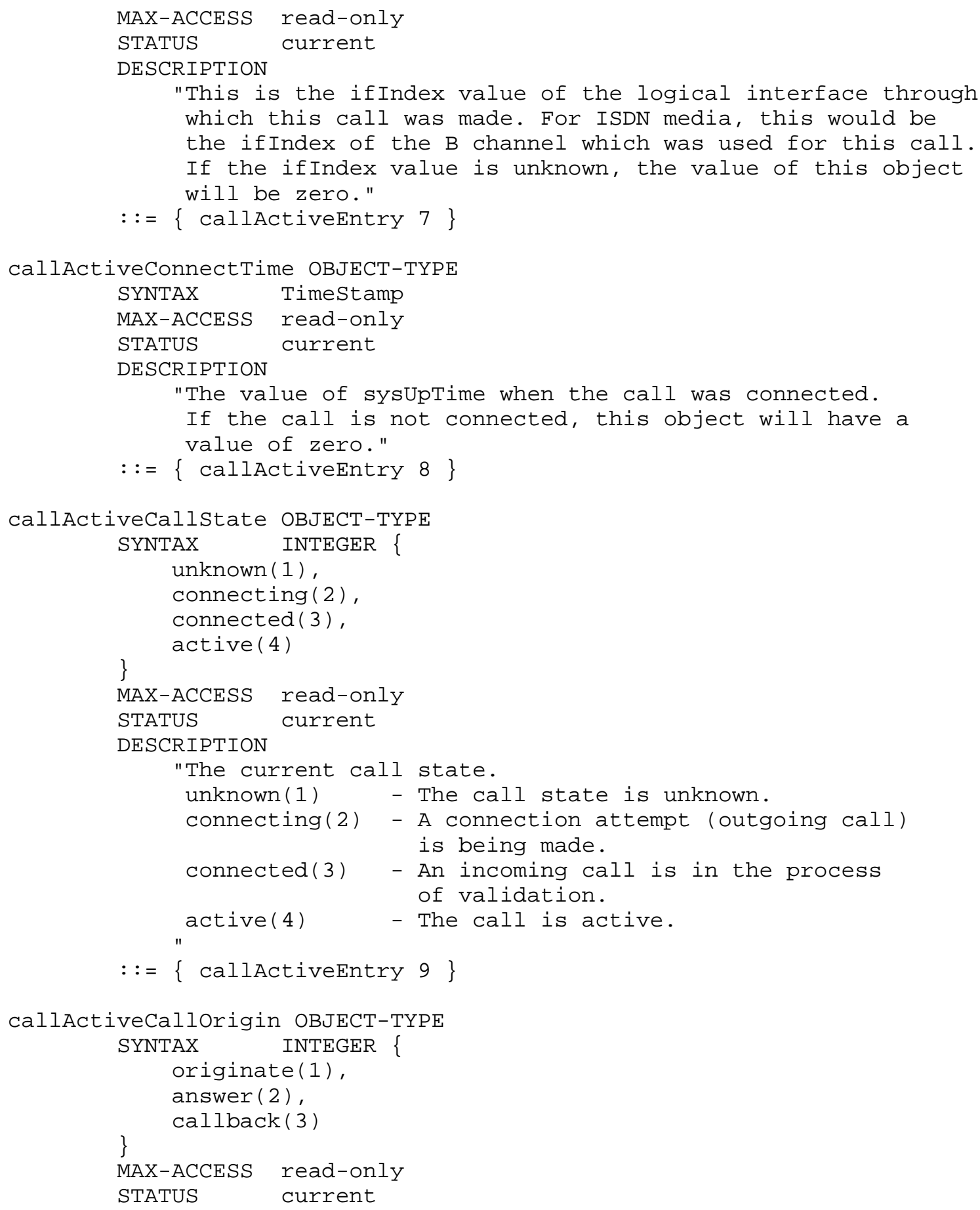




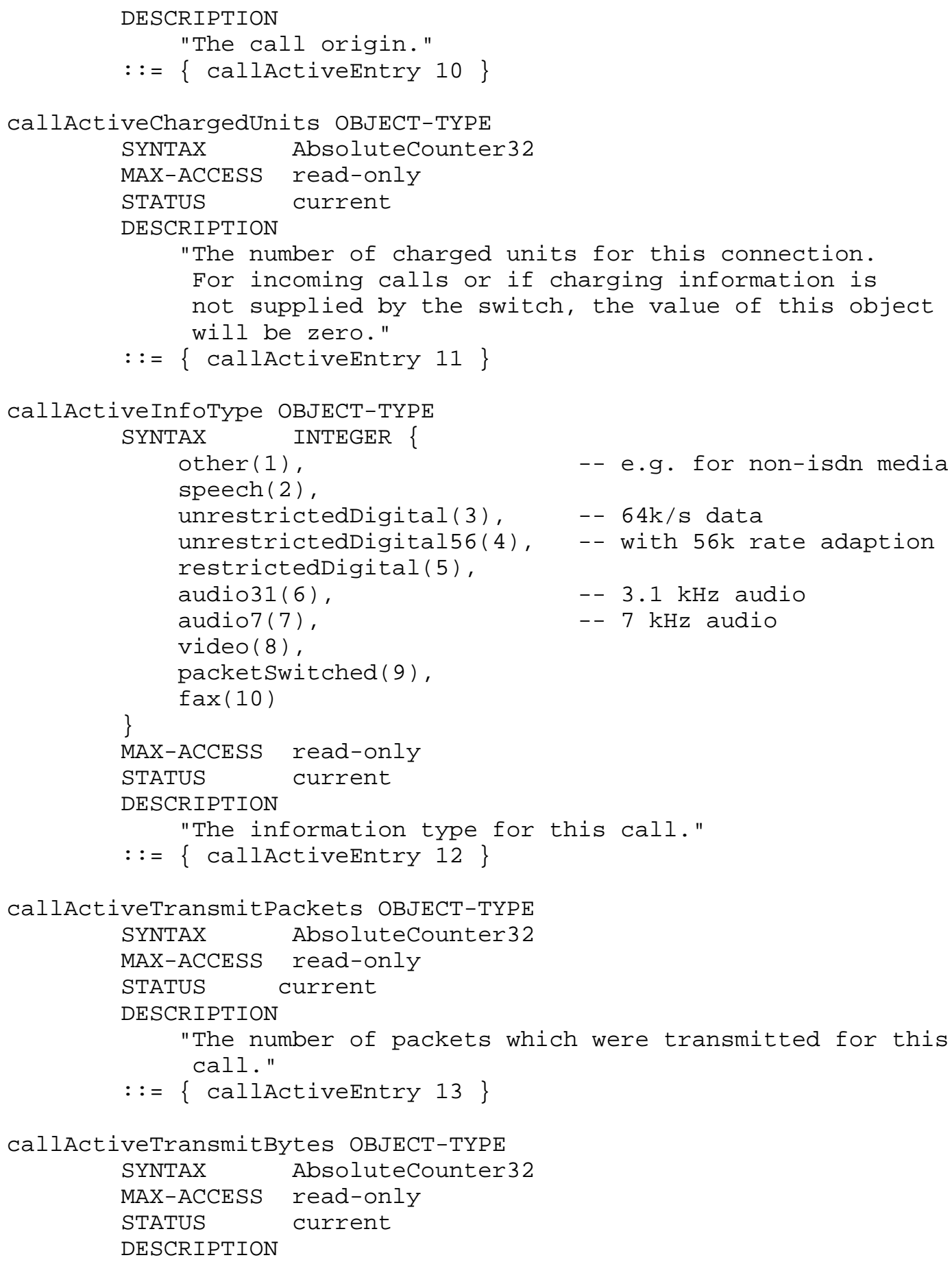




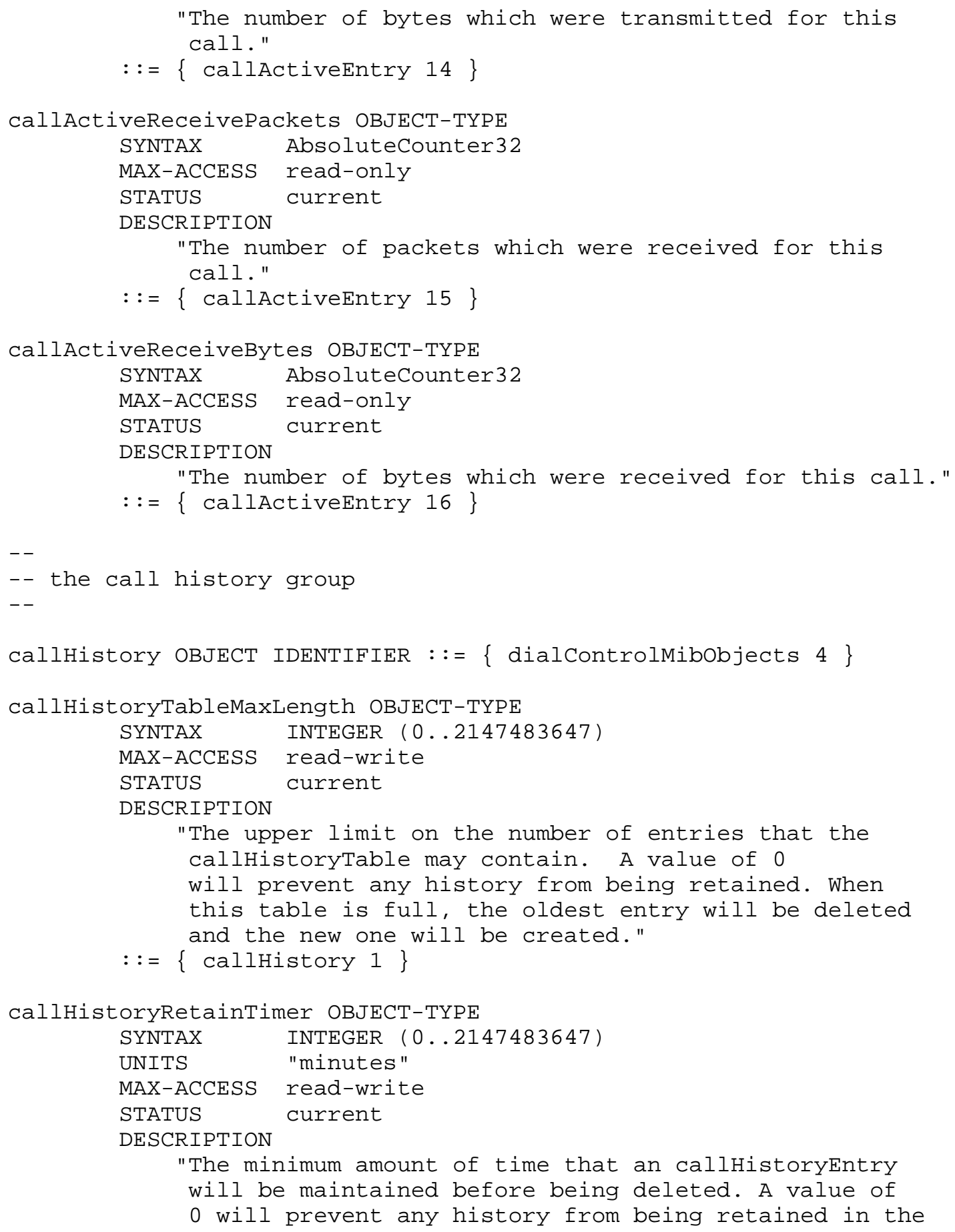

Roeck 


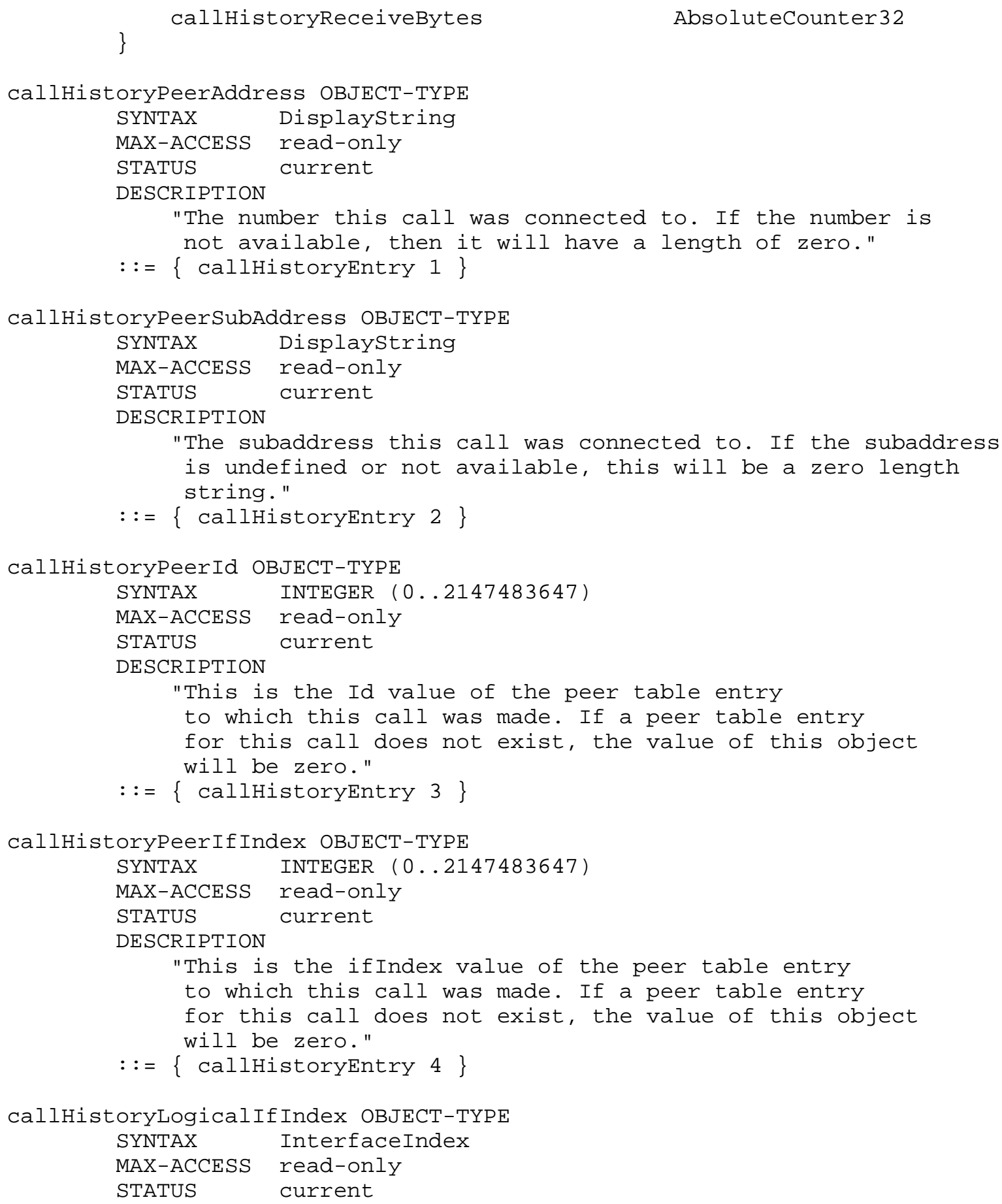

Roeck 


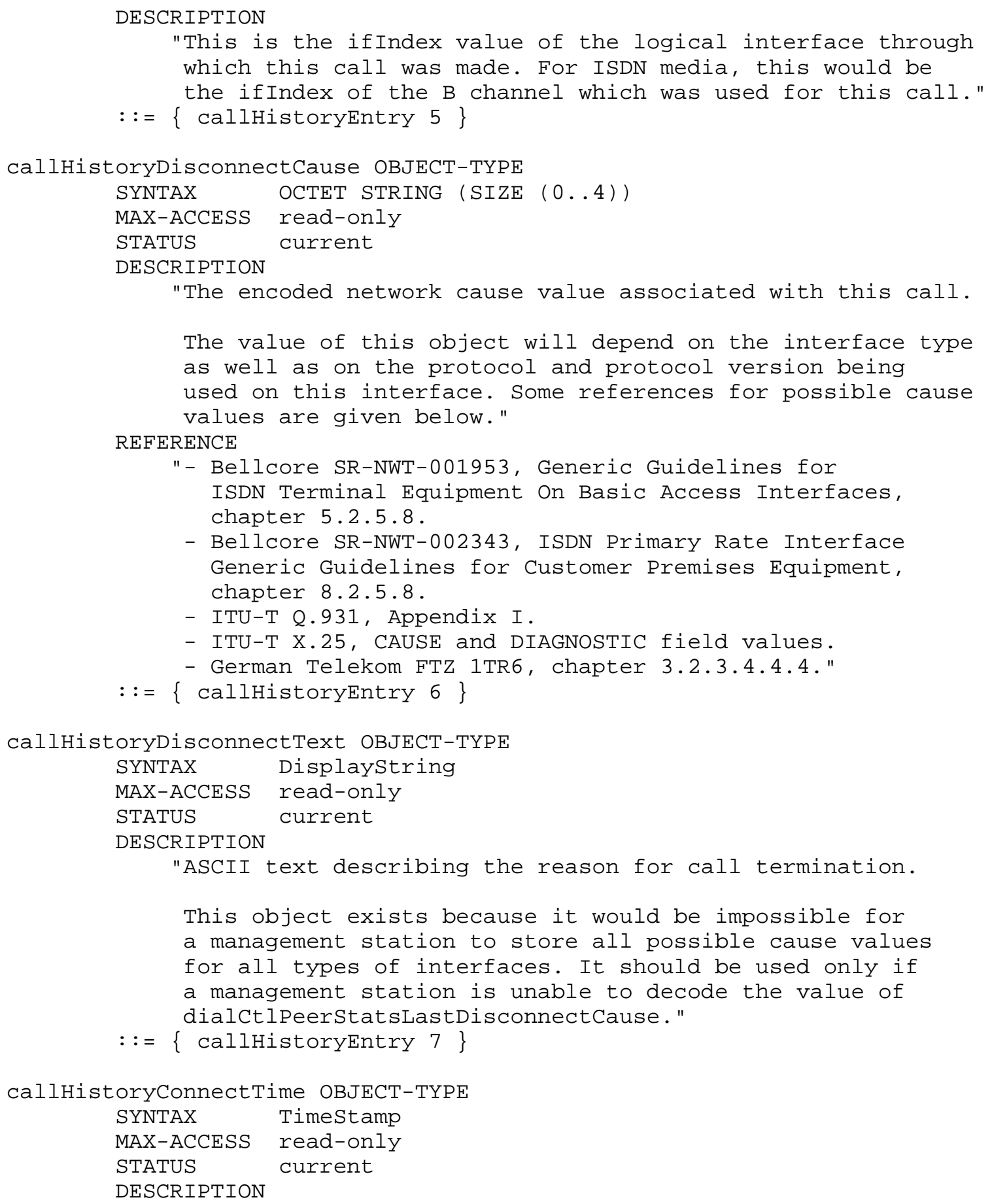




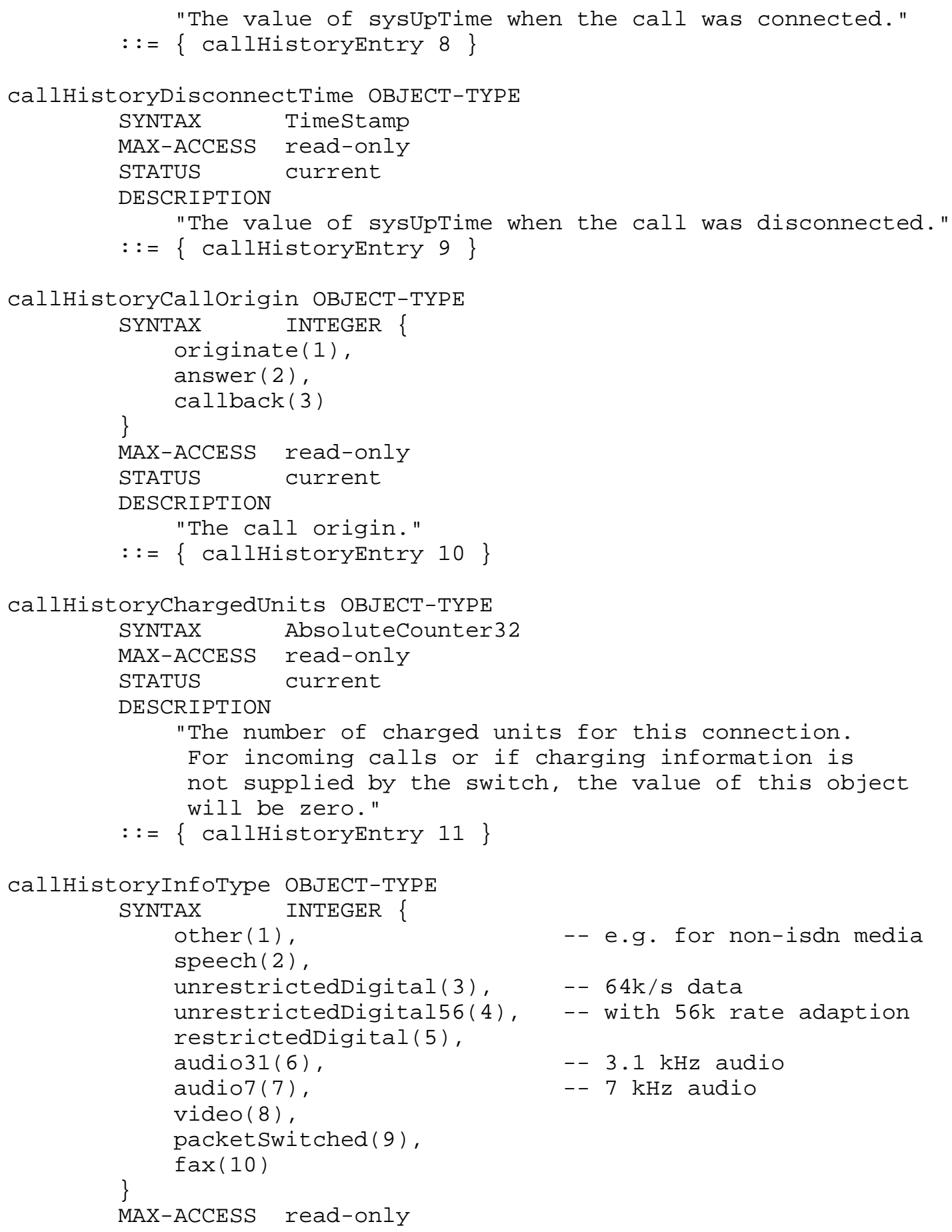




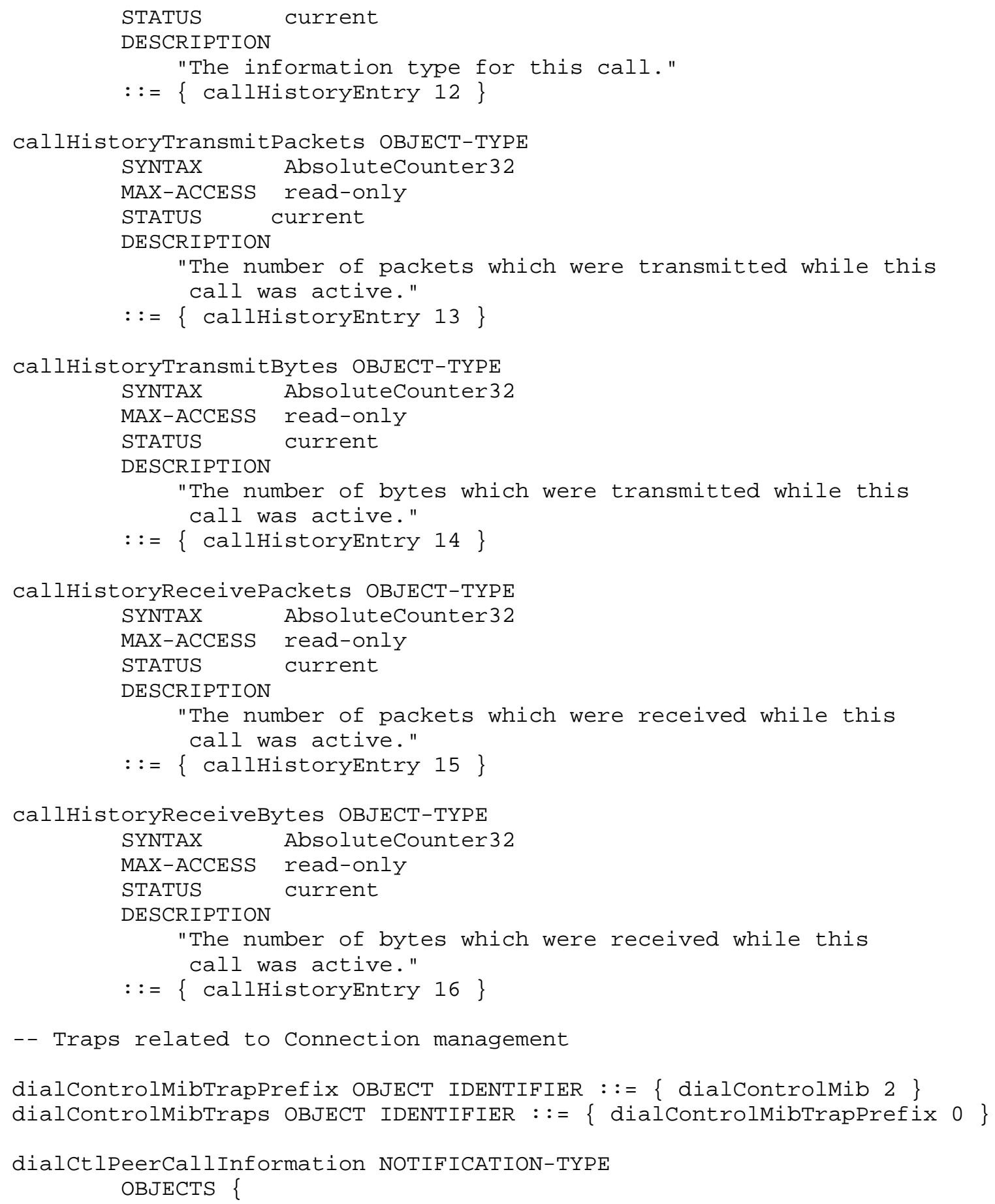

Roeck 


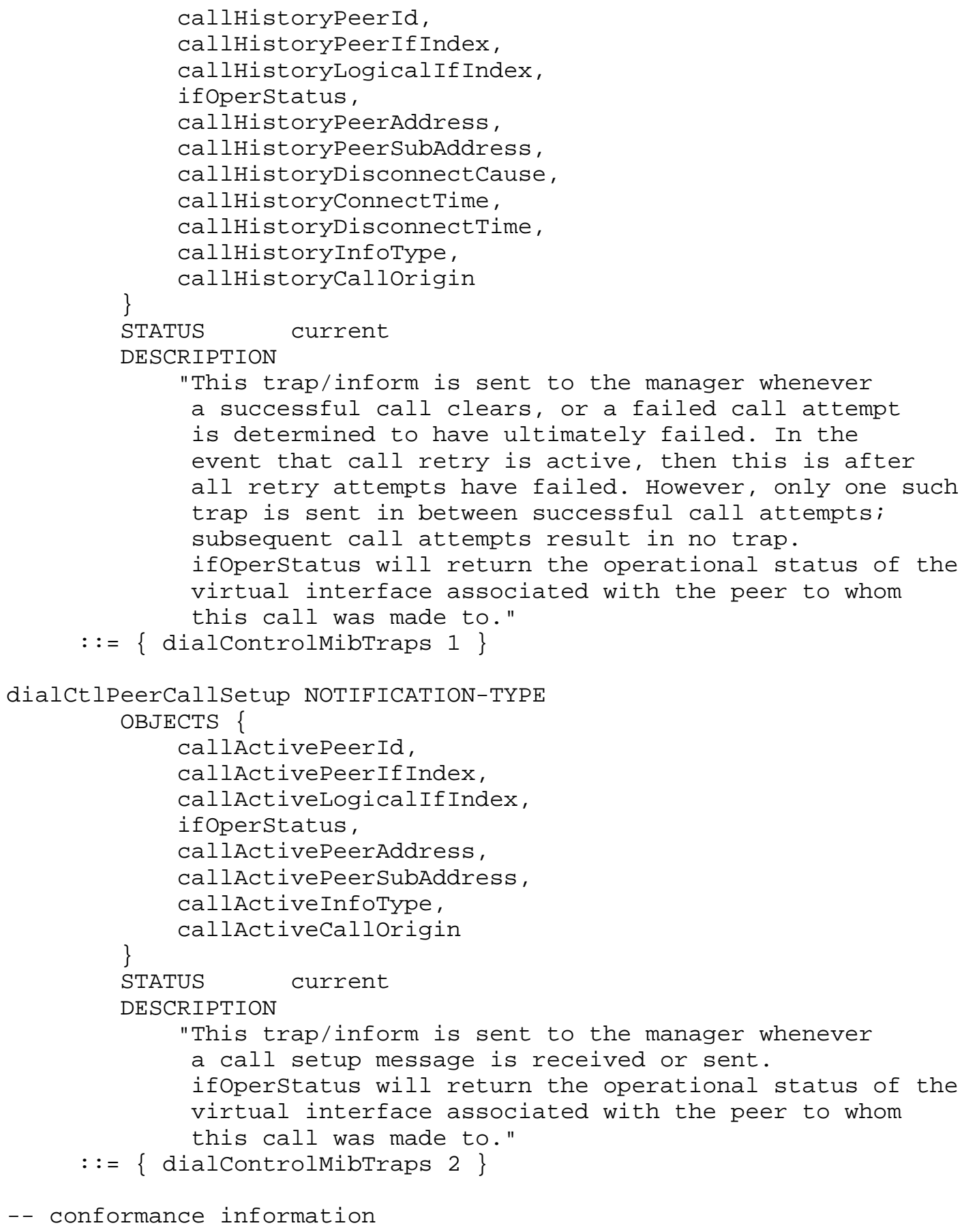

Roeck 


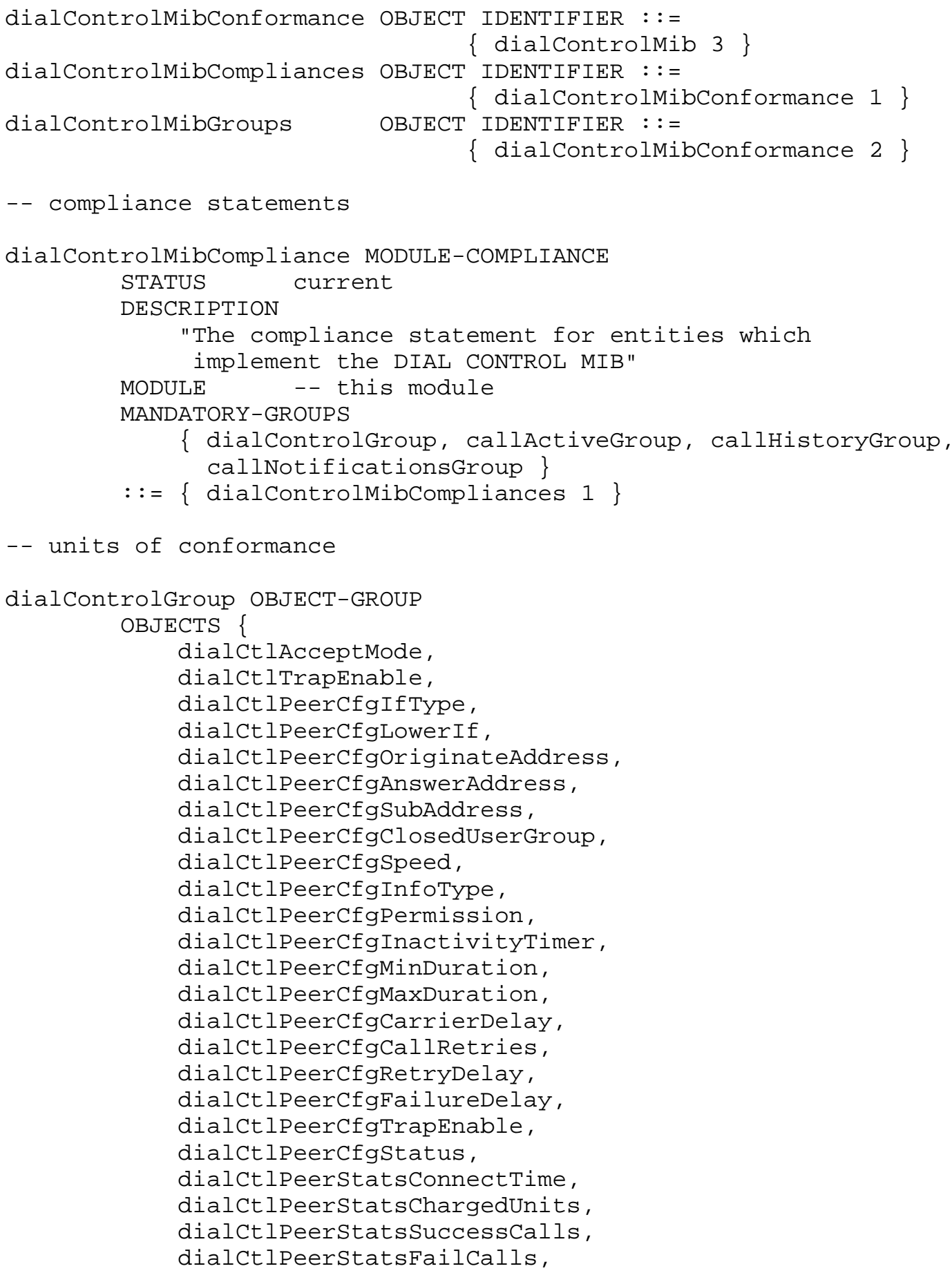

Roeck 


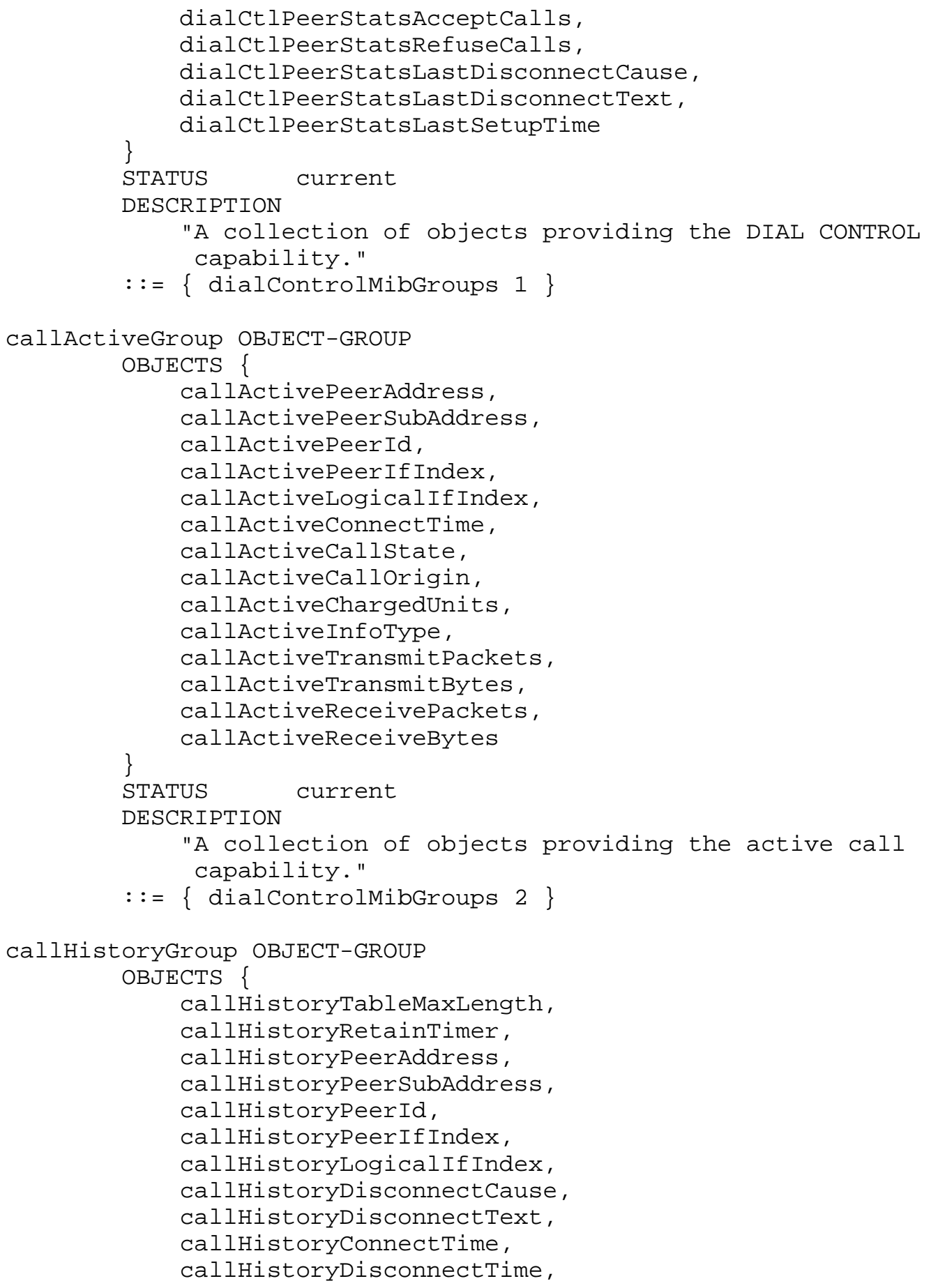

Roeck 


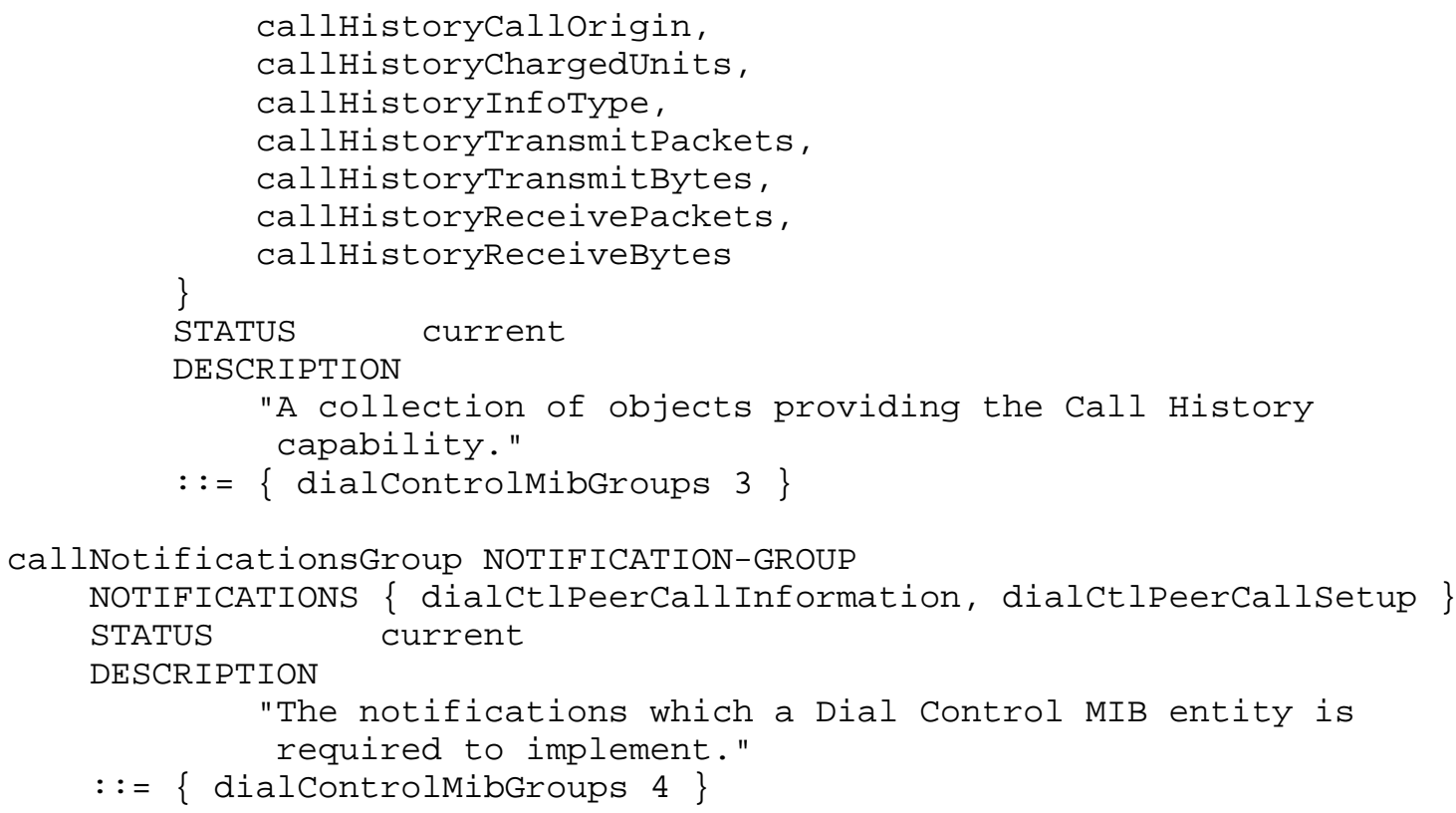

END

4. Acknowledgments

This document was produced by the ISDN MIB Working Group. Special thanks is due to the following persons:

Ed Alcoff

Fred Baker

Bibek A. Das

Ken Grigg

Jeffrey T. Johnson

Glenn Kime

Oliver Korfmacher

Kedar Madineni

Bill Miskovetz

David M. Piscitello

Lisa A. Phifer

Randy Roberts

Hascall H. Sharp

Hongchi Shih

Robert Snyder

Bob Stewart

Ron Stoughton

James Watt 


\section{References}

[1] SNMPv2 Working Group, Case, J., McCloghrie, K., Rose, M., and S. Waldbusser, "Structure of Management Information for Version 2 of the Simple Network Management Protocol (SNMPv2)", RFC 1902, January 1996 .

[2] McCloghrie, K., and M. Rose, Editors, "Management Information Base for Network Management of TCP/IP-based internets: MIB-II", STD 17, RFC 1213, Hughes LAN Systems, Performance systems International, March 1991 .

[3] Case, J., Fedor, M., Schoffstall, M., and J. Davin, "A Simple Network Management Protocol (SNMP)", STD 15, RFC 1157, SNMP Research, Performance Systems International, MIT Lab for Computer Science, May 1990.

[4] SNMPv2 Working Group, Case, J., McCloghrie, K., Rose, M. and S. Waldbusser, "Protocol Operations for Version 2 of the Simple Network Management Protocol (SNMPv2)", RFC 1905, January 1996.

[5] ITU-T Recommendation "Digital subscriber Signalling System No. 1 (DSS 1) - ISDN user-network interface layer 3 specification for basic call control", Rec. Q.931(I.451), March 1993.

[6] ITU-T Recommendation "Generic procedures for the control of ISDN supplementary services ISDN user-network interface layer 3 specification", Rec. Q.932(I.452).

[7] ITU-T Recommendation "Digital subscriber Signalling System No. 1 (DSS 1) - Signalling specification for frame-mode basic call control", Rec. Q.933.

[8] McCloghrie, K. and F. Kastenholz, "Evolution of the Interfaces Group of MIB-II", RFC 1573, Hughes LAN Systems, FTP Software, January 1994 .

6. Security Considerations

Information in this MIB may be used by upper protocol layers for security purpose.

The implementor should be aware that supporting generic peers as described in section 3.4 may cause a security hole. The user would not know where a call is from, which could potentially allow unauthorized access if there is no other authentication scheme, e.g. PPP authentication, available. 
7. Author's Address

Guenter Roeck

cisco Systems

170 West Tasman Drive

San Jose, CA 95134

U.S.A.

Phone: +1 4085273143

EMail: groeck@cisco.com 